\title{
Announcement: PRFluids publishes Invited Perspective on Grand Challenges in Environmental Fluid Mechanics
}

Environmental fluid mechanics underlies a wide range of natural, industrial, and, by extension, societal challenges. In the drive to create a more sustainable planet many problems remain outstanding, from the understanding and modeling of stratified turbulence and consequent mixing, to applied studies of pollution transport in the ocean, atmosphere, and urban environments. To encourage innovative efforts to address these problems, PRFluids has published the invited paper "Confronting Grand Challenges in environmental fluid mechanics" which summarizes the discussions and outcomes of a recent Les Houches School of Physics meeting with the intent of providing a resource for the community going forward and a plan of action for the coming decade.

(ब) Published 8 February 2021

DOI: 10.1103/PhysRevFluids.6.020001 